\section{Takotsubo cardiomyopathy: diagnosis in an emergency department}

\author{
Marina Mancini,, Davide Bartolini, ${ }^{2}$ \\ Mauro Zanna ${ }^{3}$ \\ 'Department of Emergency Medicine, \\ Villa Scassi Hospital, Local Health Unit 3; \\ 2Department of Cardiology, Villa Scassi \\ Hospital, Local Health Unit 3, Genoa, \\ Italy; ${ }^{3}$ Chief Medical Officer, Life Support \\ Camp of Al Zubair, Basrah, Iraq
}

\section{Abstract}

Takotsubo cardiomyopathy (TC) is a reversible cardiomyopathy characterized by transient wall-motion abnormalities of the left ventricle (LV) in the absence of significant obstructive coronary disease. In emergency departments the diagnosis remains a challenge because clinical and electrocardiographic presentation of Takotsubo is quite similar to ST-segment elevation myocardial infarction. We conducted a retrospective descriptive study on 1654 patients admitted to our emergency department from 2006 to 2009 who had a left heart catheterization for a suspected acute coronary syndrome and among them we evaluated characteristics on admission of 14 patients with a clinical picture suggestive for a TC. All patients were postmenopausal female. Ten patients (71\%) had preceding stressful events and four patients (29\%) did not have identifiable stressors. Thirteen patients (93\%) presented chest pain and one (7\%) syncope. ST-segment elevation was present in six patients (43\%). One patient (7\%) presented an episode of ventricular fibrillation. All patients presented increased cardiac Troponin T. Initial LV ejection fraction, evaluated by transthoracic echocardiography was $44 \pm 10 \%$. Follow-up LV ejection fraction was $61 \pm 10 \%$. Six patients (43\%) had characteristic apical ballooning and eight patients (57\%) had hypokinesia or akinesia of the apical or/and midventricular region of the $\mathrm{LV}$ without ballooning. Coronary angiography was normal in nine patients (64\%) and five (36\%) had stenosis $<50 \%$. None had complete obstruction of a coronary. Takotsubo syndrome should be considered as a possible diagnosis in patients admitted in an emergency department with a suspected diagnosis of acute coronary syndrome. Emergency physicians should recognize salient aspects of the medical history at presentation in order to organize appropriate investigations and avoid inappropriate therapies.

\section{Introduction}

Transient left ventricular apical dyskinesia, also known as Takotsubo cardiomyopathy (TC) or stress induced cardiomyopathy is a syndrome characterized by acute onset of transient extensive akinesia of some portions of the left ventricle (LV) (usually the apical and mid portion), without significant stenosis on the coronary angiography. It is often accompanied by typical chest pain, dynamic reversible ST-T segment abnormalities and increased cardiac enzymes disproportionate to the extent of akinesia.

This syndrome was first described in Japan. ${ }^{1-4}$ The name Takotsubo is explained by the LV, which, seen on echocardiography, resembles a Japanese octopus trap with a round bottom and a narrow neck. It is also called stress induced cardiomyopathy or broken heart syndrome because it is often, but not always, associated with a psychologically or stressful event.

The cause and the pathogenesis of the syndrome is still uncertain and many theories have been proposed, such as catecholaminemediated cardiotoxicity, abnormalities in coronary microvascular function and multivessel coronary vasospasm., ${ }^{5,6}$ The predominance of postmenopausal women suggests that ovarian sex hormones estrogens and progesterone can play a role in Takotsubo. Sex hormones can influence sympathetic activity and coronary reactivity and have a role in the regulation of myocardial contraction.?

The most common presenting symptom of Takotsubo is acute chest pain, but patients may also present with dyspnea, pulmonary edema and more rarely cardiogenic shock. ${ }^{2,8}$ Clinical presentation of TC mimics acute myocardial infarction (AMI) and often people presented to the emergency department with a suspected acute coronary arterial syndrome. It is impossible to distinguish between these two entities based on symptoms, electrocardiographic changes, biochemical markers or echocardiography and only after coronary angiography demonstrating no significant stenosis, diagnosis of Takotsubo syndrome can be suspected. Because the management of Takotsubo in the acute phase is different from the AMI and inadequate treatment can be dangerous for the patient, TC should be considered as an alternative diagnosis by physicians working in emergency departments.

In this report, we evaluate the clinical features of fourteen patients presented to our emergency department who underwent an urgent coronary angiography with a suspect of acute coronary arterial syndrome. The clinical picture at the end of investigation was consistent with the diagnosis of Takotsubo syndrome.
Correspondence: Marina Mancini, Department of Emergency Medicine, Villa Scassi Hospital, Local Health Unit 3, Corso Scassi 1, 16151 Genoa, Italy. Tel. +39.339.2664418 - Fax: +39.010.8492716

E-mail: dott.marina.mancini@gmail.com

Key words: Takotsubo cardiomyopathy, acute myocardial infarction, reversible left ventricular ballooning, emergency department.

Contributions: the authors contributed equally.

Conflict of interests: the authors declare no potential conflict of interests.

Received for publication: 15 February 2013 Revision received: 28 November 2013.

Accepted for publication: 20 December 2013.

This work is licensed under a Creative Commons Attribution 3.0 License (by-nc 3.0).

(C) Copyright M. Mancini et al., 2014

Licensee PAGEPress, Italy

Emergency Care Journal 2014; 10:1188

doi:10.4081/ecj.2014.1188

\section{Materials and Methods}

From May 2006 to April 2009 we retrospectively analyzed 1654 patients presented to the emergency department of Villa Scassi Hospital in Genoa, Italy, who had undergone urgent left heart catheterization immediately or within 24 hours of staying in the emergency department for a suspected acute coronary syndrome: new-onset chest pain, electrocardiographic abnormalities (ST/T wave abnormalities, abnormal $\mathrm{Q}$ waves) and/or elevated cardiac biomarkers. Among them, we identified fourteen patients with possible Takotsubo syndrome and we described their features on admission. They were included based on the following findings at cardiac catheterization or transthoracic echocardiography: i) reversible akinesia or diskinesia of the apical and/or midventricular segments of the LV with regional wall motion abnormalities; ii) absence of obstructive coronary artery disease. Clinical characteristics (age, gender, symptoms of presentation, coronary risk factors and preceding stressful events) were recorded for each patient. Cardiac biomarkers, 12 lead electrocardiographic and echocardiographic findings on admission in the emergency department were recorded. Eleven patients underwent urgent coronary angiography immediately. Three patients underwent urgent coronary catheterization after a period of 6 hours staying in our emergency department. Follow-up echocardiography was performed in twelve patients. All data are expressed as mean \pm 1 standard deviation. 


\section{Results}

Fourteen patients (100\%) were women and mean age was $67 \pm 7$ (range 48 to 80 ). Ten patients (71\%) had hypertension, six patients (43\%) were smokers, and one patient $(7 \%)$ had diabetes mellitus.

Possible precipitating factors are listed in Table 1. Ten patients (71\%) had preceding stressful events. Four patients (29\%) did not have identifiable stressors. The most common symptom of presentation was chest pain (93\%). One patient (7\%) presented with syncope.

Electrocardiographic and echocardiographic findings are presented in Table 2. Thirteen patients (93\%) had electrocardiographic abnormalities at time of presentation; one patient (7\%) had a normal electrocardiogram. ST-segment elevation was present in six patients (43\%): five (36\%) in precordial leads and one (7\%) in limb leads. One patient (7\%) with ST-segment elevation presented an episode of ventricular fibrillation. Five patients (36\%) had T-wave inversion and one (7\%) presented pathologic $Q$ waves. One patient (7\%) presented non-specific repolarization changes. Initial cardiac Troponin T was increased in all patients (100\%) on admission $(0.34 \pm 0.46$ $\mathrm{ng} / \mathrm{mL}$, normal $<0.010 \mathrm{ng} / \mathrm{mL}$ ). The initial assessment of LV ejection fraction was performed by transthoracic echocardiography. Initial LV ejection fraction was $44 \% \pm 10 \%$. Apical ballooning characteristic of Takotsubo was observed in six patients (43\%). None of the patients had midventricular ballooning or LV outflow tract obstruction. Eight patients (57\%) had hypokinesia or akinesia of the apical and/or midventricular region of the $\mathrm{LV}$ without ballooning. Left ventricular ejection fraction improved in all 12 patients who underwent follow-up transthoracic echocardiography $(61 \pm 10 \%)$. Coronary angiography was normal in nine $(64 \%)$ patients and five $(36 \%)$ had stenosis $<50 \%$. None of them needed treatment with percutaneous transluminal coronary angioplasty.

\section{Discussion}

In 1991 Sato and colleagues describe for the first time a syndrome called Takotsubo syndrome or transient cardiac ballooning syndrome. ${ }^{1}$ It is a condition characterized by transient regional systolic dysfunction of the LV associated with retrosternal pain, ST-segment elevation in the precordial leads, elevated cardiac biomarkers and no significant coronary artery disease detected on coronary angiogra- phy. Subsequently, several cases have been reported in Japan, ${ }^{2-4}$ and more recently this syndrome began to draw attention worldwide and some reports have documented its presence in other countries. ${ }^{9-11}$ Because of its clinical presentation that often mimics AMI, ${ }^{12-16}$ patients with Takotsubo are often admitted in emergency departments. Therefore, this is an important and not uncommon entity to be suspected and recognized by emergency physicians. Reported prevalence among patients with symptoms suggestive of suspected acute coronary syndrome ranges from 0.7 to $2.5 \% .^{17}$ In this single-center case series, we report on our experience of fourteen patients with clinical features suggestive for a Takotsubo syndrome including electrocardiographic abnormalities and elevated cardiac biomarkers compatible with myocardial ischemia. They represent $0.8 \%$ of patients admitted to our emergency department who had a left heart catheterization for suspected acute coronary syndrome. All patients in our series are menopausal female (mean age at presentation was $67 \pm 7$ years) and this is consistent with the published literature. ${ }^{17}$ In accordance with literature, the most frequent symptom on presentation among our patients is sudden chest pain (93\%). One patient (7\%) presented with syncope. ${ }^{8,18-20}$

A peculiar feature of this syndrome is the occurrence of an emotionally or physically stressful event before the onset of symptoms. Triggering events included emotional conditions as death of spouse, child, or close family member, domestic abuse, receiving news of serious diagnosis, public performance, losing money, loss of job, occupational stress. ${ }^{17,20}$ Reported physical stress triggers are external injury, heavy labor, travel, hip fracture, asthma, pneumothorax, cerebrovascular accident and intense physical exercise..$^{10,17,20}$ However, in $30 \%$ there was no preceding emotional or physical stressful event identified. In the present analysis, a stressor event was identifiable in ten patients (71\%).

Reported cardiovascular risk factors associated to Takotsubo are arterial hypertension, diabetes mellitus, dyslipidemia and current or past smoking. ${ }^{2,4,17}$ In our report, ten patients (71\%) have a history of hypertension, six (46\%) are current or past smokers, two (15\%) have diabetes and two (15\%) have dyslipidemia.

On presentation, electrocardiographic characteristics of Takotsubo are similar to that observed in patients with myocardial infarction. The most frequent findings on the admission are ST-segment elevation in precordial leads which occurs in approximately 50 to $60 \%$ of patients and T-waves inversion in most leads. ${ }^{2,8,9,16,19}$ In our patients, ST-segment elevation was present in $46 \%$ and T-wave inversion in $38 \%$ of patients. One patient (7\%) developed

Table 1. Triggers of Takotsubo.

Type of stress

Physical (intense physical exercise, trauma, renal colic)

Emotional (death of family member, quarrelling, receiving news of husband's serious diagnosis, financial instability, husband's stroke)

Unidentified

Table 2. Electrocardiographic and echocardiographic parameters on admission.

\begin{tabular}{lc} 
Variable & Value (\%) \\
ECG & \\
ST-segment elevation & $6(43)$ \\
T-wave inversion & $5(36)$ \\
Non specific repolarization changes & $1(7)$ \\
Pathologic Q waves & $1(7)$ \\
Right bundle branch & $1(7)$ \\
First degree atrioventricular block & $1(7)$ \\
Prolonged corrected QT interval in lead V3 & $1(7)$ \\
Ventricular fibrillation & $1(7)$ \\
Echo & \\
Initial LV ejection fraction (\%) & $44 \pm 10$ \\
Presence of apical ventricular ballooning & $6(43)$ \\
Presence of midventricular ballooning & 0 \\
LV outflow tract obstruction & 0 \\
\hline
\end{tabular}

ECG, electrocardiography; Echo, echocardiography; LV, left ventricle. No. of patients 4 6 4 
pathologic Q-wave in anteroseptal leads; one patient $(7 \%)$ presented non-specific repolarization changes. Previous reports describe that the corrected QT interval can be temporarily prolonged, ${ }^{13,14}$ but life-threatening arrhythmias are uncommon despite marked, structural abnormalities. ${ }^{21}$ Corrected QT interval was prolonged only in one (7\%) of our patients. One patient admitted to our emergency department with chest pain and a slight ST-segment elevation in anteroseptal leads (Figure 1) during the medical examination presented an episode of ventricular fibrillation (Figure 2). Defibrillation with 200 Joule was given and the rhythm reverted to normal sinus rhythm after the first shock.

Although there are reports about the possibility to distinguish Takotsubo from anterior AMI, ${ }^{13,14,22-24}$ electrocardiographic changes themselves do not have sufficient predictive value for discriminating Takotsubo from AMI. Neither cardiac enzymes are helpful: some patients have no rise in cardiac biomarkers, but most have a slight elevation, usually disproportionate to the extent of akinesia of the $\mathrm{LV}^{25}$ In our study all patients showed a widely variable elevation of troponin $\mathrm{T}$.

The echocardiographic findings in Takotsubo are more typical: akinesia of the LV apex, hypercontraction of the basal segments and midventricular hypokinesia causing apical ballooning and reduced LV ejection fraction (20-40\%). The wall motion abnormality typically extends beyond the distribution of any one coronary vessel. The apical ballooning is rapidly reversible and normalization of the echocardiogram is usually seen within four to eight weeks. ${ }^{4,8}$ The baseline LV ejection fraction in our population is $44 \pm 10 \%$ and the typical apical ballooning was present in $43 \%$ of our patients. Of the fourteen patients, twelve had follow-up ejection fraction determination and all of them had an improvement in their LV ejection fraction $(61 \pm 10 \%)$. There are reports about Takotsubo variants in which the middle segments of the ventricle balloons during systole, called midventricular ballooning, inverted Takotsubo pattern or non apical ballooning. ${ }^{26}$ None of our patients shows non apical ballooning.

On the basis of demographic and clinical features, electrocardiographic and echocardiographic findings and laboratory abnormalities, emergency physicians should consider a possible diagnosis of Takotsubo. Nevertheless, in order to definitely distinguishing Takotsubo from an AMI, urgent coronary angiography is necessary. Typically, patients with stress induced cardiomyopathy have normal coronary arteries or occasionally mild irregularities. ${ }^{15}$ In accordance, nine (64\%) of our patients have normal coronary angiography and five (36\%) had $<50 \%$ stenosis.

The diagnostic criteria for Takotsubo remain controversial. The proposed modified Mayo Clinic criteria ${ }^{16}$ for the clinical diagnosis of Takotsubo are shown in Table 3. These criteria are entirely satisfied by our patients. It is to note that the possibility that patients with obstructive coronary atherosclerosis develop Takotsubo has been recently reported. Therefore some authors proposed that the second Mayo Clinic diagnostic criterion be replaced with a large discrepancy between dyskinetic segments and coronary lesions on the angiogram, either qualitative (different vascular territories) or quantitative (segmental extension). ${ }^{27}$ Others suggested changing the second criterion to a nonobstructive coronary disease of less than $50 \%$ stenosis. ${ }^{28}$

Because the pathophysiology is unclear and major prospective randomized controlled trials are absent, optimal treatment for Takotsubo

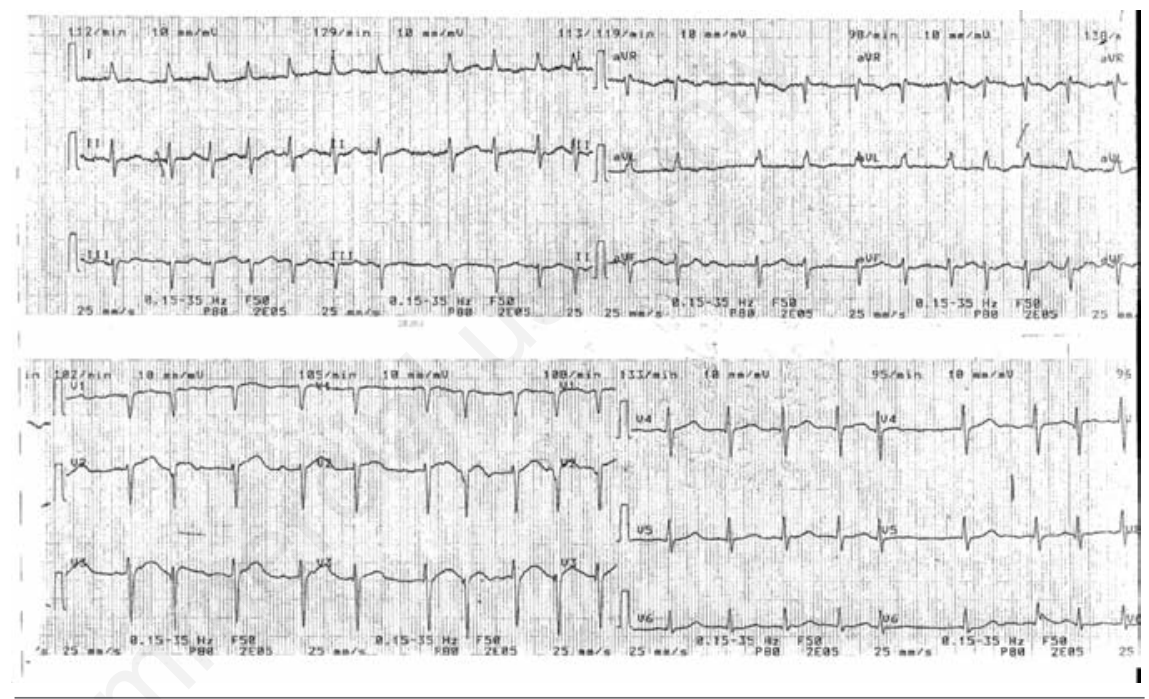

Figure 1. Twelve-leads electrocardiography in a patient on admission.

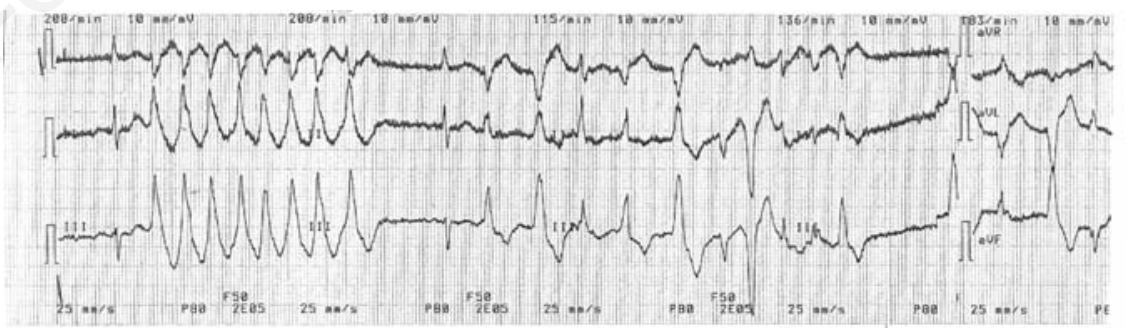

Figure 2. Episode of ventricular fibrillation reverted to sinus rhythm after a 200 Joule shock.

Table 3. Proposed Mayo Clinic criteria for the clinical diagnosis of Takotsubo cardiomyopathy.

Criteria

Reversible LV dysfunction, with RWMA not confined to a particular epicardial coronary artery irrigation area ${ }^{\circ}$

Exclusion of angiographic evidence of obstructive coronary artery disease, or ruptured plaque

New ECG abnormalities and/or elevated troponin

Absence of recent head trauma, intracranial hemorrhage, pheochromocytoma, myocarditis, hypertrophic cardiomyopathy

LV, left ventricle; RWMA, regional wall motion abnormality; ECG, electrocardiography. ${ }^{\circ}$ However, some patients can have atypical Takotsubo cardiomyopathy with RWMA confined to a single coronary area; therefore the judgement requires additional factors indicated as in rows below. 
has yet to be established. Therefore, at present, treatment is mainly symptomatic and supportive. Because the presentation is indistinguishable from an acute coronary syndrome, in an emergency department the treatment should be directed toward the myocardial ischemia. Aspirin, cardioselective b-blockers and ACE-inhibitors have been used during the LV dysfunction. b-blockers were also suggested as treatment to prevent malignant arrhythmias. If $\mathrm{LV}$ outflow tract obstruction is a predominant feature intravenous fluid with short acting b-blockers should be cautiously administered to decrease contractility and increase LV cavity size. Severe cardiogenic shock should be treated with an intra-aortic balloon pump or inotropic support. ${ }^{20}$

The overall prognosis is favorable. The most frequent complication is left-sided heart failure. In the acute phase, deaths due to cardiogenic shock, ventricular fibrillation, ventricular rupture, mitral valve dysfunction and pulmonary embolism are reported. Reported recurrence rate of Takotsubo is very low..$^{20}$

\section{Conclusions}

The frequency of the diagnosis of TC has increased over the past few years, perhaps due to increasing awareness among cardiologists. In the most of cases, emergency physicians are the first to manage with these patients and thus it is an important entity they have to know and to understand, especially at present when the emergency physician is increasingly taking much more autonomy in diagnostic and therapeutic management of patients.

At initial presentation the diagnosis of Takotsubo syndrome remains a challenge because of the similarity between this syndrome and that of ST elevation myocardial infarction (STEMI). It is a necessary recognition of salient aspects of the medical history and a high index of suspicion for thinking about stress induced cardiomyopathy.

At presentation symptoms, electrocardiographic features and cardiac enzymes may not be conclusive. An urgent transthoracic echocardiography can be performed and the feature of a characteristic apical ballooning can be useful for the diagnosis, but the association of Takotsubo with contraction patterns not strictly conforming to the original description make it more difficult to diagnose. The suspicion of Takotsubo can be confirmed, in most of cases, by an urgent coronarography. It is important differentiating Takotsubo from an AMI, not only because the outcome is different, but also for avoiding inadequate treatment in the acute phase. This is crucial for emergency physicians working in an institution without primary percutaneous coronary intervention and for physicians working in prehospital setting, when fibrinolytic therapy is considered for a possible STEMI because inappropriate administration of fibrinolytics to a patient with Takotsubo may lead to potential heavy risks as bleeding complications, acute renal failure and others.

Cardiovascular magnetic resonance can have an additional role in recognizing this entity also in acute phase and recent technical advances in magnetic resonance imaging will lead to better diagnostic accuracy in evaluating patients with chest pain presenting to emergency department. ${ }^{29}$ However, at present TC remains a diagnosis of exclusion that can be only suspected on presentation in an emergency department and may be confirmed by a repetitive echocardiographic evaluation showing complete normalization of the contractile abnormalities.

\section{References}

1. Dote K, Sato H, Tateishi $\mathrm{H}$, et al. Myocardial stunning due to simultaneous multivessel coronary spasms: a review of 5 cases. J Cardiol 1991;21:203-14.

2. Tsuchihashi K, Ueshima K, Uchida T, et al. Transient left ventricular apical ballooning without coronary artery stenosis: a novel heart syndrome mimicking acute myocardial infarction. J Am Coll Cardiol 2001;38: 11-8.

3. Kurisu S, Sato H, Kawagoe T, et al. Takotsubo-like left ventricular dysfunction with ST-segment elevation: a novel cardiac syndrome mimicking acute myocardial infarction. Am Heart J 2002;143:448-55.

4. Abe Y, Kondo M, Matsuoka R, et al. Assessment of clinical features in transient left ventricular apical ballooning. J Am Coll Cardiol 2003;41:737-42.

5. Wittstein IS, Thiemann DR, Lima JA, et al. Neurohumoral features of myocardial stunning due to sudden emotional stress. New Engl J Med 2005;352:539-48.

6. Nef HM, Mollmann H, Akashi YJ, et al. Mechanisms of stress (Takotsubo) cardiomyopathy. Nat Rev Cardiol 2010;7:187-93.

7. Kuo BT, Choubey R, Novaro GM. Reduced estrogens in menopause may predispose women to takotsubo cardiomyopathy. Gender Med 2010;7:71-7.

8. Bybee KA, Kara T, Prasad A, et al. Systematic review: transient left ventricular apical ballooning: a syndrome that mimics ST-segment elevation myocardial infarction. Ann Intern Med 2004;141:85865.

9. Desmet WJR, Adriaenssens BFM, Dens JAY. Apical ballooning of the left ventricle: first series in white patients. Heart
2003;89:1027-31.

10. Bybee KA, Prasad A, Barsness GW, et al. Clinical characteristics and thrombolysis in myocardial infarction frame counts in women with transient left ventricular apical ballooning syndrome. Am J Cardiol 2004;94:343-6.

11. Sharkey SW, Lesser JR, Zenovich AG, et al. Acute and reversible cardiomyopathy provoked by stress in women from the United States. Circulation 2005;111:472-9.

12. Abe Y, Kondo M. Apical ballooning of the left ventricle: a distinct entity? Heart 2003;89:974-6.

13. Ogura R, Hiasa Y, Takahashi T, et al. Specific findings of the standard 12-lead ECG in patients with "Takotsubo" cardiomyopathy: comparison with the findings of acute anterior myocardial infarction. Circ J 2003;67:687-90.

14. Kurisu S, Inoue I, Kawagoe T, et al. Time course of electrocardiographic changes in patients with Tako-tsubo syndrome: comparison with acute myocardial infarction with minimal enzymatic release. Circ J 2004;68:77-81.

15. Prasad A. Apical ballooning syndrome: an important differential diagnosis of acute myocardial infarction. Circulation 2007;115:e56-9.

16. Prasad A, Lerman A, Rihal CS. Apical ballooning syndrome (Tako-tsubo or stress cardiomyopathy): a mimic of acute myocardial infarction. Am Heart J 2008;155: 408-17.

17. Vizzardi E, D’Aloia A, Zanini G, et al. Takotsubo-like left ventricular dysfunction: transient left ventricular apical ballooning syndrome. Int J Clin Pract 2010;64:67-74.

18. Pillière R, Mansencal N, Digne F, et al. Prevalence of tako-tsubo syndrome in a large urban agglomeration. Am J Cardiol 2006;98:662-5

19. Matsuoka K, Okubo S, Fujii E, et al. Evaluation of the arrhythmogenicity of stress-induced "takotsubo cardiomyopathy" from the time course of the 12-lead surface electrocardiogram. Am J Cardiol 2003;92:230-3.

20. Parodi G, Del Pace S, Carrabba N, et al. Incidence, clinical findings, and outcome of women with left ventricular apical ballooning syndrome. Am J Cardiol 2007;99:182-5.

21. Dib C, Prasad A, Friedman PA, et al. Malignant arrhythmia in apical ballooning syndrome: risk factors and outcomes. Indian Pacing Electrophysiol J 2008;8:18292.

22. Inoue $\mathrm{M}$, Shimizu $\mathrm{M}$, Ino $\mathrm{H}$, et al. Differentiation between patients with Takotsubo cardiomyopathy and those with anterior acute myocardial infarction. Circ J 2005;69:89-94. 
23. Bybee KA, Motiei A, Syed IS, et al. Electrocardiography cannot reliably differentiate transient left ventricular apical ballooning syndrome from anterior STsegment elevation myocardial infarction. J Electrocardiol 2007;40:38.

24. Jim MH, Chan A0, Tsui PT, et al. A new ECG criterion to identify Takotsubo cardiomyopathy from anterior myocardial infarction: role of inferior leads. Heart Vessels 2009;24:124-30.

25. Sharkey SW, Lesser JR, Menon M, et al. Spectrum and significance of electrocardiographic patterns, troponin levels, and thrombolysis in myocardial infarction frame count in patients with stress (takotsubo) cardiomyopathy and comparison to those in patients with ST-elevation anterior wall myocardial infarction. Am J Cardiol 2008;101:1723-8.

26. Song BG, Chun WJ, Park YH, et al. The clinical characteristics, laboratory parameters, electrocardiographic, and echocardiographic findings of reverse or inverted takotsubo cardiomyopathy: comparison with mid or apical variant. Clin Cardiol 2011;34:693-9.

27. Gaibazzi N, Ugo F, Vignali L, et al. Tako- tsubo cardiomyopathy with coronary artery stenosis: a case-series challenging the original definition. Int J Cardiol 2009;133: 205-12.

28. Nussinovitch U, Goitein 0, Nussinovitch $\mathrm{N}$, et al. Distinguishing a heart attack from the "broken heart syndrome" (Takotsubo Cardiomyopathy). J Cardiovasc Nurs 2011; 26:524-9.

29. Assomull RG, Lyne JC, Keenan N, et al. The role of cardiovascular magnetic resonance in patients presenting with chest pain, raised troponin, and unobstructed coronary arteries. Eur Heart J 2007;28:1242-9. 